\section{EL OCIO COMO VALOR EN LA SOCIEDAD ACTUAL}

\author{
Iratxe Aristegui Fradua \\ María Silvestre Cabrera \\ Universidad de Deusto \\ Avenida de las Universidades, 24 \\ 48007, Bilbao \\ iariste@deusto.es \\ maria.silvestre@deusto.es
}

\begin{abstract}
Leisure is an unquestionable value in today's society. In this article we will try to reflect the increasing importance that the free time and leisure are having in our life, based on the results obtained in the European values survey in its implementation in Europe, Spain and the Basque country. It will try to show a comparative overview of the three realities identified, the importance and the meaning of leisure, over time, in a period that ranges from 1999 until 2008.
\end{abstract}

KEY WORDS: Values; leisure; free time; work. Social context; evolution.

\section{INTRODUCCIÓN}

El tiempo de ocio juega un papel cada vez más importante en las vidas de las personas $y$, sobre todo, en la valoración que éstas le atribuyen. De hecho, el ocio parece ser un valor cada vez más preciado en nuestra sociedad, junto a cuestiones como la familia, el trabajo o las amistades. Esta es la razón por la que hemos creído de interés analizar el valor que adquiere el tiempo de ocio (y el tiempo libre) en las sociedades actuales, analizando qué importancia se le concede y qué significado le otorgamos.

La información utilizada para esta aproximación proviene de la Encuesta Europea de Valores en sus aplicaciones a Europa, España y al País Vasco en 1999 y 2008. El hecho de aplicarse en diferentes países al mismo tiempo nos posibilita establecer comparaciones con otros entornos. Esto quiere decir que el análisis que aqui se presenta combina una perspectiva longitudinal a la vez que comparada.

Existe un previo metodológico que nos condiciona tanto analítica como teóricamente. Nos referimos al hecho de trabajar

\section{LEISURE AS A VALUE IN TODAY'S SOCIETY}

RESUMEN: El ocio representa un valor indiscutible en la sociedad actual. En este artículo vamos a tratar de reflejar la cada vez mayor importancia que el tiempo libre y de ocio están adquiriendo en nuestra vida, a partir de los resultados obtenidos en la Encuesta Europea de Valores en su aplicación en Europa, España y País Vasco. Se tratará de mostrar una visión comparativa de las tres realidades señaladas en cuanto a la importancia y el significado del ocio, a lo largo del tiempo, en un período que comprende desde 1999 hasta 2008.

PALABRAS CLAVE: Valores; ocio; tiempo libre; trabajo; contexto social; evolución.

con una encuesta que aborda diversas cuestiones valorativas (relativas a familia, trabajo, religión, medio ambiente, política, economía, ocio, etc.) pero que no se centra exclusivamente en analizar el ocio, sino que lo recoge como una cuestión más y plantea las preguntas sin hacer grandes distinciones entre el el ocio y el tiempo libre, conceptos que son utilizados como sinónimos'. Entendemos que este hecho puede suponer una limitación a la hora de interpretar los datos; sin embargo, consideramos que se trata de una base de datos que, a pesar de sus limitaciones, ofrece grandes ventajas. La principal, la posibilidad de comparar el valor atribuido al ocio por la sociedad europea en su conjunto y, a su vez, la bondad de poder contrastar cómo ha evolucionado esta valoración y percepción a lo largo de los últimos diez años.

También queremos destacar que cuando se llevó a cabo el trabajo de campo (verano de 2008) la crisis económica mundial todavía no era demasiado palpable, por lo que hay que entender los resultados en un contexto de bonanza económica en el cual probablemente el valor Trabajo pierda parte de su centralidad a favor de otros valores como, por ejemplo, el Ocio. 


\section{IMPORTANCIA DEL OCIO EN LA VIDA DE LAS PERSONAS}

En la siguiente tabla mostramos la comparación entre los distintos aspectos sobre los que se les ha preguntado a la población tanto en 1999 como en 2008 para ofrecer un repaso sobre su evolución con el paso del tiempo.
Atendiendo a la evolución de los distintos aspectos en los dos períodos objeto de estudio se puede observar en general $y$, sobre todo, teniendo en cuenta la suma de las dos opciones:

- La indiscutible primacía de la Familia como valor fundamental de nuestras vidas,

TABLA 1. GRADO DE IMPORTANCIA QUE TIENE EN SU VIDA CADA UNO DE LOS SIGUIENTES ASPECTOS TENIENDO EN CUENTA LAS OPCIONES "MUY IMPORTANTE" Y "BASTANTE IMPORTANTE" (\% HORIZONTALES)

\begin{tabular}{|c|c|c|c|c|c|c|c|}
\hline & \multicolumn{2}{|c|}{ Muy importante } & \multicolumn{2}{|c|}{ Bastante importante } & \multicolumn{2}{|c|}{ Suma de las dos } & \multirow{2}{*}{$\begin{array}{c}\text { Diferencia } \\
2008-1999\end{array}$} \\
\hline & 1999 & 2008 & 1999 & 2008 & 1999 & 2008 & \\
\hline \multicolumn{8}{|l|}{ EUROPA } \\
\hline Familia & 84,3 & 84 & 13,3 & 14,1 & 97,6 & 98,2 & 0,60 \\
\hline Trabajo & 58 & 58,1 & 32,2 & 31,3 & 90,2 & 89,4 & $-0,80$ \\
\hline Amigos & 40,2 & 41,7 & 48,2 & 47,6 & 90,4 & 89,3 & $-1,10$ \\
\hline Tiempo libre/de ocio & 32 & 34,5 & 49,3 & 49,7 & 81,3 & 83,2 & $+1,90$ \\
\hline Religión & 20,4 & 27,7 & 29,7 & 32,7 & 50,1 & 60,4 & 10,30 \\
\hline Politica & 7,3 & 9,3 & 26,5 & 26,7 & 33,8 & 35,0 & 1,20 \\
\hline \multicolumn{8}{|l|}{ ESPAÑA } \\
\hline Familia & 85,7 & 83,2 & 13,2 & 15,5 & 98,9 & 98,7 & $-0,20$ \\
\hline Trabajo & 63 & 61,8 & 31,6 & 30,0 & 94,6 & 91,8 & $-2,80$ \\
\hline Amigos & 39,4 & 42,1 & 47,2 & 49,2 & 86,6 & 91,3 & 4,70 \\
\hline Tiempo libre/de ocio & 31,5 & 40,9 & 49,4 & 49,9 & 80,9 & 90,8 & $+9,90$ \\
\hline Religión & 14,9 & 14,1 & 27,1 & 22,5 & 42,0 & 36,6 & $-5,40$ \\
\hline Politica & 4,4 & 5,5 & 14,9 & 21,1 & 19,3 & 26,6 & 7,30 \\
\hline \multicolumn{8}{|l|}{ PAÍS VASCO } \\
\hline Familia & 85,6 & 88,8 & 13,2 & 10,3 & 98,8 & 99,1 & 0,30 \\
\hline Trabajo & 62,1 & 60,3 & 30,5 & 35,1 & 92,6 & 95,4 & $+2,80$ \\
\hline Amigos & 54,7 & 57,1 & 40,6 & 36,6 & 95,3 & 93,4 & $-1,90$ \\
\hline Tiempo libre/de ocio & 47,8 & 56,2 & 43,9 & 39,7 & 91,7 & 95,9 & $+4,20$ \\
\hline Religión & 14 & 13,7 & 29,7 & 19,5 & 43,7 & 32,6 & $-11,10$ \\
\hline Politica & 3,8 & 7,9 & 18,7 & 20,4 & 22,5 & 27,5 & 5,00 \\
\hline
\end{tabular}

Fuente: Elaboración propia a partir de las encuestas de valores europea, española y vasca (1999 y 2008).

- la creciente ascensión de la importancia del Tiempo libre/ de Ocio tanto en Europa como en el País Vasco y, sobre todo, en España donde sube casi 10 puntos,

- la notable aproximación entre las valoraciones del Trabajo y del Tiempo libre/de Ocio (ver gráfico), destacando como segundo valor más importante -después de la familia e incluso por encima del trabajo-, en el caso vasco.

Asistimos, por tanto, a una cierta pérdida de peso del Trabajo y a una revalorización del Tiempo libre/de Ocio; o 
TABLA 2. COMPARACIÓN DE LA IMPORTANCIA CONCEDIDA AL TRABAJO Y AL OCIO EN 1999 Y 2008 (\%)

\begin{tabular}{|l|c|c|c|c|}
\hline \multirow{2}{*}{} & \multicolumn{2}{|c|}{$\begin{array}{c}\text { TRABAJO MUY + BASTANTE } \\
\text { IMPORTANTE }\end{array}$} & \multicolumn{2}{c|}{$\begin{array}{c}\text { OCIO MUY + BASTANTE } \\
\text { IMPORTANTE }\end{array}$} \\
\cline { 2 - 5 } & $\mathbf{1 9 9 9}$ & $\mathbf{2 0 0 8}$ & $\mathbf{1 9 9 9}$ & $\mathbf{2 0 0 8}$ \\
\hline EUROPA & 90,2 & 89,4 & 81,3 & 83,2 \\
\hline ESPAÑA & 94,6 & 91,8 & 80,9 & 90,8 \\
\hline PAíS VASCO & 92,6 & 95,4 & 91,7 & 95,9 \\
\hline
\end{tabular}

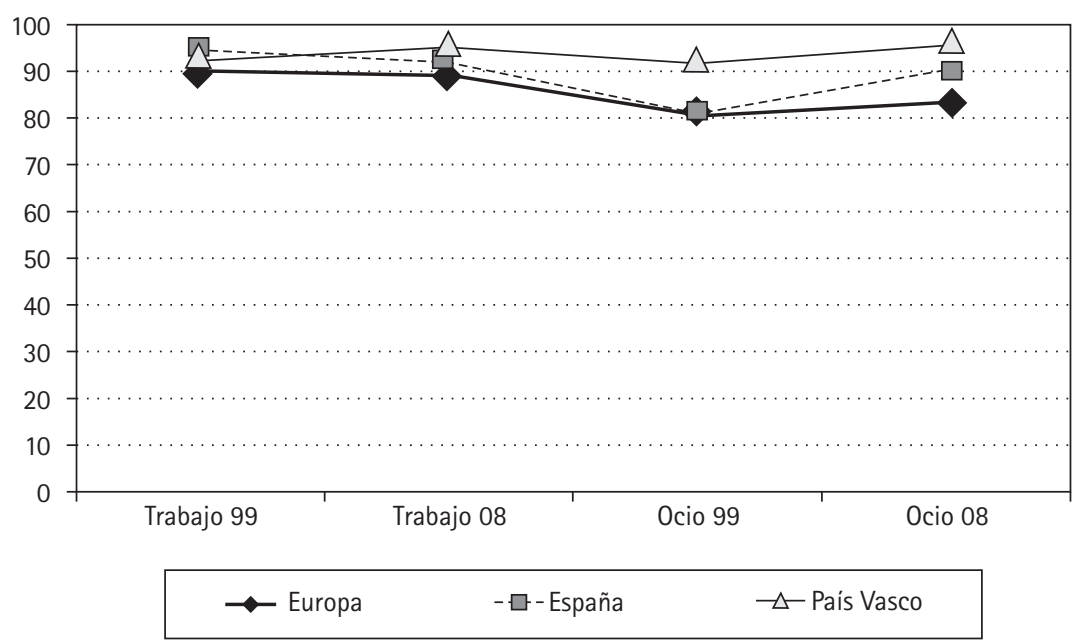

Fuente: Elaboración propia a partir de las encuestas de valores europea, española y vasca (1999 y 2008).

lo que es lo mismo, al "casi perfecto" equilibrio en la balanza Trabajo-Tiempo libre/de Ocio, hasta ahora inclinada a favor del Trabajo. Parece, como señaló C. Offe (1992) hace ya algunos años, que el trabajo está dejando de ser una categoría social clave en nuestra sociedad (Offe, C., 1997, 36; Gorz, A., 1997). 0 que, tal y como considera uno de los pioneros de la Sociología del Ocio, Dumazedier, en la sociedad moderna industrial, el Tiempo libre/de Ocio ha pasado a ser una esfera cada vez más significativa de la vida; esto es, "se ha afirmado no sólo como una atractiva posibilidad, sino como un valor" (Dumazedier, J., 1964, 22).

Del mismo modo, como puede apreciarse en la tabla 3, si consideramos sólo la opción "muy importante", y hacemos una comparación en cuanto al Trabajo y el Tiempo libre/ de 0 cio, vemos que la tendencia de una evolución lineal en ambos casos (una decreciente y la otra creciente) es bastante clara. Así, mientras que el Trabajo se mantiene o va perdiendo algo de importancia entre 1999 y 2008, el Tiempo libre/de Ocio va ganando terreno de manera progresiva, sobre todo, en lo que respecta a España y, más aún, al País Vasco.

Esta tendencia hacia una creciente importancia del tiempo libre -más acusada en España y en el País Vasco que en Europa en general- parece confirmarse también, en el hecho de que la población vasca está más en desacuerdo que de acuerdo con que "el trabajo es siempre lo primero aunque ello signifique menos tiempo libre". Por otra parte, aunque en España todavía se le sigue otorgando una gran importancia al trabajo $(51,8 \%)$, también es cierto que el desacuerdo con esta afirmación es mayor que en 1999 (31\% frente a $25 \%$ ) (ver tabla 4).

ARBOR Vol. 188754 marzo-abril [2012] 283-291 ISSN: 0210-1963 
TABLA 3. COMPARACIÓN DE LA IMPORTANCIA CONCEDIDA AL TRABAJO Y AL OCIO TENIENDO EN CUENTA LA OPCIÓN "MUY IMPORTANTE" (\%)

\begin{tabular}{|l|c|c|c|c|}
\hline \multirow{2}{*}{} & \multicolumn{2}{|c|}{ TRABAJO MUY IMPORTANTE } & \multicolumn{2}{c|}{ OCIO MUY IMPORTANTE } \\
\cline { 2 - 5 } & $\mathbf{1 9 9 9}$ & $\mathbf{2 0 0 8}$ & $\mathbf{1 9 9 9}$ & $\mathbf{2 0 0 8}$ \\
\hline EUROPA & 58,0 & 58,1 & 32,0 & 34,5 \\
\hline ESPAÑA & 63,0 & 61,8 & 31,5 & 40,9 \\
\hline PAÍS VASCO & 62,1 & 60,3 & 47,8 & 56,2 \\
\hline
\end{tabular}

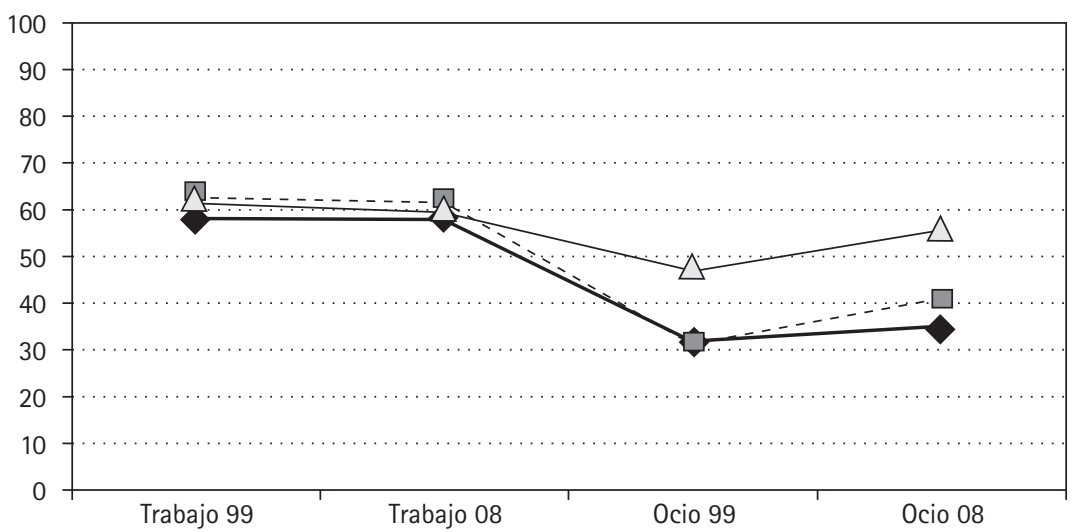

$\rightarrow$ Europa $\quad-\square$-España $\quad \triangle$ Pais Vasco

Fuente: Elaboración propia a partir de las encuestas de valores europea, española y vasca (1999 y 2008).

TABLA 4. ACUERDO CON QUE EL TRABAJO ES SIEMPRE LO PRIMERO (\% HORIZONTALES)

\begin{tabular}{|l|c|c|c|c|c|c|c|}
\hline \multirow{2}{*}{} & & \multicolumn{3}{|c|}{ ESPAÑA } & \multicolumn{3}{c|}{ PAíS VASCO } \\
\cline { 3 - 9 } & & Acuerdo & Desac. & Dif. A-D & Acuerdo & Desac. & Dif. A-D \\
\hline $\begin{array}{l}\text { El trabajo es siempre lo primero aunque signifique menos } \\
\text { tiempo libre }\end{array}$ & $\mathbf{1 9 9 9}$ & 50,8 & 24,7 & $+26,1$ & 48,4 & 33 & $+15,4$ \\
\cline { 3 - 9 } & $\mathbf{2 0 0 8}$ & 51,8 & 31,1 & $+20,7$ & 39,9 & 45,1 & $-5,2$ \\
\hline
\end{tabular}

Fuente: Elaboración propia a partir de las encuestas de valores española y vasca (1999 y 2008).

Esto quiere decir, que cada vez nos inclinamos más a valorar un trabajo pero con el condicionante de que no nos reste tiempo libre.

Sin embargo, tampoco queremos obviar que todavía son muy significativos los niveles de acuerdo con el hecho de que el trabajo "sea siempre lo primero". Esta cuestión, que sí ha sido objeto de lenta y constante evolución en los últimos diez años, es un tema que bien puede ponerse en relación con el debate suscitado en España, y también en el País Vasco, sobre la necesidad de racionalizar los tiempos de trabajo productivo para acercarlos a los modelos horarios europeos, donde existe una división mucho más clara entre los tiempos del trabajo productivo y los tiempos para la vida personal, familiar y de ocio. Modelos que son más productivos y que permiten una mejor conciliación. 


\subsection{La importancia del ocio en función de distintas variables sociodemográficas}

En el apartado anterior hemos reflejado la presencia del Ocio como un valor en alza en la sociedad actual. Ahora bien, si consideramos algunos de los distintos países en los que se ha suministrado la encuesta europea, observamos claramente que la variable "país" influye de manera considerable en la opinión que la ciudadanía tiene sobre este aspecto.

TABLA 5. GRADO DE IMPORTANCIA QUE SE CONCEDE AL TIEMPO LIBRE O DE OCIO SEGÚN EL PAIS DE PROCEDENCIA. \% HORIZONTALES (ORDENADAS SEGÚN LAS MEDIAS), 2008

\begin{tabular}{|l|c|c|c|c|}
\hline & Muy importante & Bastante importante & Suma de las dos & MEDIAS * \\
\hline EUROPA 2008 & 34,5 & 49,7 & 83,2 & $\mathbf{1 , 8 3}$ \\
\hline PAÍS VASCO & 56,2 & 39,7 & 95,9 & $\mathbf{1 , 4 8}$ \\
\hline HOLANDA & 52,3 & 42,9 & 95,2 & $\mathbf{1 , 5 3}$ \\
\hline IRLANDA & 52,9 & 41,0 & 93,9 & $\mathbf{1 , 5 4}$ \\
\hline GRECIA & 46,6 & 48,7 & 95,3 & $\mathbf{1 , 5 8}$ \\
\hline MALTA & 48,0 & 43,1 & 91,1 & $\mathbf{1 , 6 2}$ \\
\hline ESPAÑA & 40,9 & 49,9 & 90,8 & $\mathbf{1 , 7 0}$ \\
\hline FRANCIA & 33,3 & 52,1 & 85,4 & $\mathbf{1 , 8 3}$ \\
\hline ALEMANIA & 30,7 & 54,5 & 85,2 & $\mathbf{1 , 8 5}$ \\
\hline BULGARIA & 26,8 & 55,8 & 82,6 & $\mathbf{1 , 9 3}$ \\
\hline PORTUGAL & 22,1 & 60,6 & 82,7 & $\mathbf{1 , 9 7}$ \\
\hline KOSOVO & 29,2 & 39,9 & 69,1 & $\mathbf{2 , 0 5}$ \\
\hline LITUANIA & 19,2 & 58,0 & $\mathbf{7 1 , 2}$ & $\mathbf{2 , 0 6}$ \\
\hline ALBANIA & 15,3 & 50,7 & $\mathbf{2 , 2 5}$ \\
\hline
\end{tabular}

En una escala del 1 (muy importante) a 4 (nada importante). *Cuanto más se acercan las puntuaciones medias al valor 1 mayor importancia le están concediendo a la variable analizada (Ocio).

Fuente: Elaboración propia a partir de la encuesta de valores europea (2008).

Vemos así, que en países como Holanda o Irlanda, incluso en el País Vasco, más de mitad de la población consultada considera el Ocio como un aspecto "muy importante" de su vida, mientras que en países como Albania, Lituania, Kosovo, Portugal, Bulgaria o incluso Alemania, por el contrario, la opción "muy importante" no es respaldada ni por un tercio de la población (véase el gráfico de la página siguiente).

El diferente peso valorativo que obtiene el tiempo de ocio en los diferentes paises donde se ha aplicado la Encuesta Europea de Valores pone de manifiesto que los diferentes modelos sociopolíticos $y$, sobre todo, los diferentes momentos en los modelos de crecimiento económico condicionan la importancia atribuida al valor ocio/tiempo libre ya que la mayoría de la ciudadanía establece una relación, bien latente, bien manifiesta, entre el tiempo de ocio y el resto de los tiempos de su vida, sin percibir los inevitables encuentros y solapamientos que se superponen en la gestión de estos tiempos (Veira, 2004).

Por otro lado, centrándonos ahora en el caso concreto de la población vasca, entre quienes mayoritariamente parecen apoyar esta opción se encuentran los hombres más que 


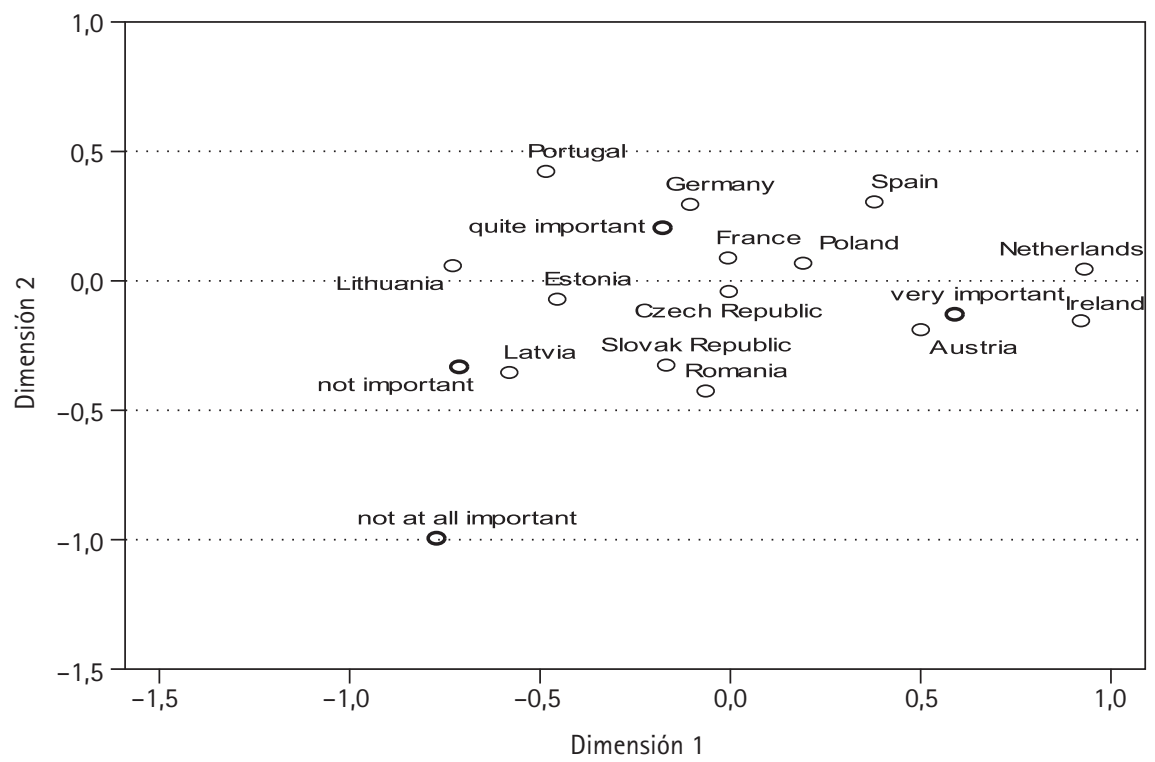

country code

how important in your life: leisure time (Q1D)

Fuente: Elaboración propia a partir de la base de datos de valores europea, 2008.

las mujeres; las personas menores de 54 años; quienes poseen más estudios; los y las estudiantes; quienes tienen un trabajo autónomo y el personal asalariado con jornadas superiores a las 30 horas.

Estos datos reflejan de manera clara que al referirse al tiempo de Ocio, la ciudadanía lo identifica más como tiempo Libre -el que les gustaría disponer- fuera del trabajo o de las horas de estudio, que con el Ocio en sí mismo. Así, los que menos lo demandan son los que, en principio, más tiempo libre parecen tener (mayores, desempleados, amas de casa...). Podría decirse, por tanto, que entendemos el significado de tiempo libre o de ocio en contraposición al tiempo dedicado al trabajo remunerado. Seguimos pensando $u$ organizando nuestra vida en torno al esquema trabajo/tiempo libre/ocio.

La diferencia que se registra entre mujeres y hombres es de tan sólo tres puntos porcentuales, es decir, los hombres valoran tres puntos más que las mujeres el ocio en sus vidas. Otras encuestas, como las Encuestas del Uso del Tiempo, recogen el dato de que los hombres dedican más horas a la semana al ocio que las mujeres. Es posible que la diferencia en la atribución de importancia esté altamente relacionada con el hecho de que los hombres disponen de más tiempo que las mujeres para disfrutar del ocio, por lo que podemos trabajar con la hipótesis de que ellos valorarian un tiempo ya invertido en ocio - pero fuera del trabajo habitual- y ellas un deseo de tiempo para invertir en ocio.

Sin embargo, no es el sexo la variable más explicativa de la distinta importancia atribuida al ocio. La edad, nivel de estudios o situación profesional influyen de forma más significativa en dicha atribución.

Como puede verse en la tabla 6, la relación del ocio con la edad es inversamente proporcional; esto quiere decir que, "a más edad menor importancia otorgada al ocio, mientras que a menos edad mayor importancia del ocio". Así, a partir de los 55 años $y$, sobre todo, a partir de los 65 años, las personas encuestadas manifiestan un menor interés por el tiempo de ocio, probablemente porque perciban que tienen 
TABLA 6. VALORACIÓN DEL OCIO COMO MUY IMPORTANTE (2008)

\begin{tabular}{|c|c|}
\hline PAÍS VASCO & 56,2 \\
\hline \multicolumn{2}{|l|}{ SEXO } \\
\hline Hombre & 57,7 \\
\hline Mujer & 54,7 \\
\hline \multicolumn{2}{|l|}{ EDAD } \\
\hline $18-24$ & 65,2 \\
\hline $25-34$ & 63,0 \\
\hline $35-44$ & 63,1 \\
\hline $45-54$ & 61,4 \\
\hline $55-64$ & 44,1 \\
\hline 65 y más & 40,6 \\
\hline \multicolumn{2}{|l|}{ NIVEL DE ESTUDIOS } \\
\hline Primarios & 40,0 \\
\hline Secundarios & 58,7 \\
\hline Superiores & 68,2 \\
\hline \multicolumn{2}{|l|}{ SITUACIÓN ACTUAL/OCUPACIÓN } \\
\hline Trabaja 30 horas semanales o más & 60,2 \\
\hline Trabaja menos de 30 horas & 52,6 \\
\hline Trabajador autónomo & 69,7 \\
\hline Retirado/pensionista & 52,2 \\
\hline Ama de casa, no empleada de otra forma & 40,6 \\
\hline Estudiante & 77,3 \\
\hline Desempleado & 45,8 \\
\hline
\end{tabular}

Fuente: Elaboración propia a partir de la base de datos de valores de Euskadi, 2008. suficiente tiempo libre o le den mayor importancia a otros valores. Esta diferencia puede ser explicada también a partir de los diferentes modelos de socialización de estas generaciones y de aprehensión de valores como el trabajo y el esfuerzo.

Por otro lado, la mayoría de las personas encuestadas, como veremos más adelante, no relacionan el ocio con tiempos de crecimiento personal o como tiempos de producción. Esta explicación bien puede valer para entender porqué quienes tienen estudios superiores valoran más el ocio. Esta es otra de las variables que más inciden en la respuesta puesto que, como muestran los datos, las personas con estudios superiores son las que más importancia otorgan al ocio, suponiendo casi treinta puntos porcentuales más que las personas con estudios primarios. De nuevo aquí, la atribución diferenciada de significado al ocio puede ser la explicación última de la diferente valoración. En el siguiente apartado podremos contrastar esta opinión puesto que abordamos el significado del tiempo libre/de ocio.

\subsection{Significado del tiempo libre/tiempo de ocio}

Antes de proceder a interpretar el significado atribuido al tiempo de ocio, debemos mencionar que partimos de las definiciones utilizadas por el cuestionario de la encuesta europea de valores. Este cuestionario limita la definición a las siguientes opciones: "relajarse", "conocer gente agradable", "hacer lo que yo quiera" y "aprender algo". Estas cuatro definiciones se refieren a cuatro dimensiones o formas de entender el tiempo libre/de ocio: el ocio como

TABLA 7. ASPECTOS SOBRE EL TIEMPO DE OCIO QUE SON IMPORTANTES PARA LA GENTE (\%) 2008

\begin{tabular}{|c|c|c|c|c|c|c|c|}
\hline \multirow{3}{*}{ Relajarse } & & \multicolumn{2}{|c|}{ EUROPA } & \multicolumn{2}{|c|}{ ESPAÑA } & \multicolumn{2}{|c|}{ PAÍS VASCO } \\
\hline & Muy importante & 52,1 & & 56,4 & & 66,9 & \\
\hline & Bastante importante & 41,9 & 94 & 38,8 & 95,2 & 29,2 & 90,1 \\
\hline \multirow{2}{*}{ Conocer gente agradable } & Muy importante & 48,1 & \multirow{2}{*}{92,7} & 38,6 & \multirow{2}{*}{88,9} & 53,0 & \multirow{2}{*}{94,4} \\
\hline & Bastante importante & 44,6 & & 50,3 & & 41,4 & \\
\hline \multirow{2}{*}{ Hacer lo que yo quiera } & Muy importante & 47,2 & \multirow{2}{*}{90,5} & 43,5 & \multirow{2}{*}{88,2} & 53,0 & \multirow{2}{*}{91,3} \\
\hline & Bastante importante & 43,3 & & 44,7 & & 38,3 & \\
\hline \multirow{2}{*}{ Aprender algo nuevo } & Muy importante & 40,7 & \multirow{2}{*}{84,9} & 36,8 & \multirow{2}{*}{84,4} & 48,4 & \multirow{2}{*}{90,8} \\
\hline & Bastante importante & 44,2 & & 47,6 & & 42,4 & \\
\hline
\end{tabular}

Fuente: Elaboración propia a partir de la base de datos de valores europea, española y vasca, 2008. 

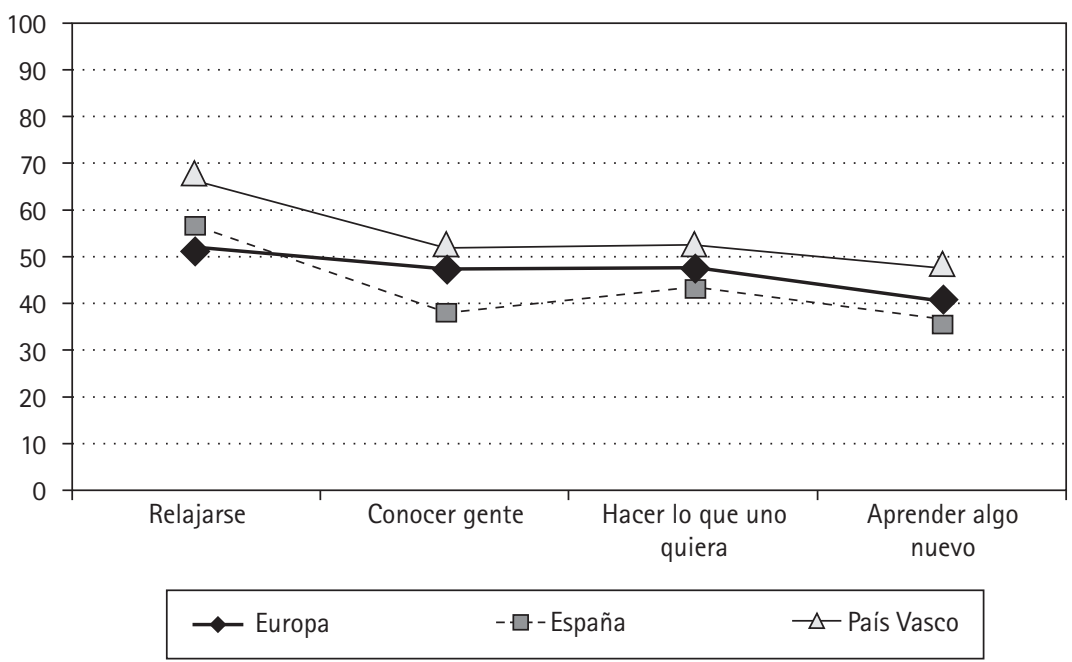

Fuente: Elaboración propia a partir de la base de datos de valores europea, española y vasca, 2008.

momento de descanso y desconexión, claramente contrapuesta a otras actividades que supongan mayor actividad; el ocio vinculado a las relaciones sociales; el ocio asociado a un comportamiento individualista y más aislado, liberado de controles jerárquicos $y$, por último; el ocio como un tiempo también productivo y de enriquecimiento personal a través de la formación y aprendizaje.

Si analizamos los datos, observamos que el aspecto con el que más se asocia la idea del ocio, tanto en Europa como en España y el País Vasco, es con la idea de la relajación, descanso o desconexión, mientras que la opción más activa y productiva, vinculada al aprendizaje, es la que menos se vincula a la idea del ocio. Y probablemente sea ésta última la más relacionada con aquellas personas que poseen estudios superiores.

\section{Conclusiones y Consideraciones finales}

El análisis e interpretación de los datos de la Encuesta Europea de Valores en su aplicación en Europa, España y País Vasco con relación a la importancia y significado atribuido al ocio, entendido casi como un sinónimo del tiempo libre, nos indica que existe una significativa relación entre la vivencia y percepción del trabajo remunerado y la significación e importancia otorgada al ocio.

En la sociedad actual, el tiempo libre/de ocio ha pasado a ser una esfera cada vez más significativa de la vida afirmándose no sólo como una atractiva posibilidad, sino como un valor en sí mismo, mientras que el trabajo ha ido perdiendo parte de su centralidad sobre todo en el período estudiado (1999-2008).

Los diferentes patrones culturales $y$, sobre todo, los diferentes niveles de crecimiento económico y bienestar sociopolítico enfatizan esta relación latente, a nivel valorativo, entre trabajo y ocio. Así, en aquellos países europeos con menores niveles de bienestar, la valoración en positivo del ocio disminuye y se incrementa el valor trabajo y a la inversa. Esta relación bien pudiera ponerse en relación tanto con los valores materialistas y postmaterialistas y con las teorias sobre las jerarquias valorativas, estableciendo la hipótesis de que si contraponemos trabajo versus ocio, podríamos estar enfrentando un valor materialista ante un valor postmaterialista respectivamente, al margen de la significación atribuida a ambos.

La mayor importancia asignada al ocio proviene de las personas más jóvenes y con estudios superiores. Este dato 
nos indica que no sólo el contexto socioeconómico explica la valoración otorgada al ocio, ya que también inciden los procesos de socialización y los valores aprehendidos. Sin duda, las nuevas generaciones y, sobre todo, quienes tienen estudios secundarios y superiores, valoran en mayor medida el ocio, posiblemente porque le atribuyen una diferente significación y definición.

De hecho, hemos visto como de entre los cuatro posibles significados del ocio, el que lo define como "relajarse" es el más generalizado, siendo la definición asociada al aprendizaje y crecimiento personal la que menos se menciona.
Si nos centramos en el País Vasco, observamos cómo también se establece la relación entre el valor trabajo y el valor ocio, y cómo el valor atribuido al ocio ha ido creciendo paulatinamente y cómo ha disminuido la importancia otorgada al trabajo, sin que éste haya dejado de ser importante.

Por último, no queremos dejar de realizar una reflexión en torno a la significación que las personas encuestadas muestran ante el tiempo de ocio, en el sentido de que todavía no existe una percepción del tiempo de ocio como un tiempo que puede solaparse con otros tiempos, un tiempo que puede ser activo, en suma, un tiempo que no debería enfrentarse, necesariamente, al tiempo productivo.

\section{NOTA}

Recibido: 17 de mayo de 2011 Aceptado: 15 de julio de 2011
1 El cuestionario de la Encuesta Europea de Valores recoge la siguiente indicación para el encuestador: "Defina tipo de ocio: tiempo no transcurrido en el trabajo remunerado o en tareas domésticas".

\section{BIBLIOGRAFÍA}

Andres Orizo, F. y Elzo, J. (dirs.) (2000): España 2000, entre el localismo y la globalidad. La encuesta europea de valores en su tercera aplicación, 19811999, Madrid, Ediciones SM.

Aristegui, I. (2010): "El valor del trabajo", en Elzo, J. y Silvestre, M. (dirs.), Hacia un individualismo protegido y placentero: cuarta encuesta europea de valores en su aplicación a España, Bilbao, Universidad de Deusto.
Dumazadier, J. (1964): Hacia una civilización del ocio, Barcelona, Espelta.

Gorz, A. (1997): Metamorfosis del trabajo Madrid, Sistema.

Offe, C. (1992): La sociedad del trabajo, Madrid, Alianza Editorial.

Offe, C. (1997): ¿Qué crisis? Retos y transformaciones de la sociedad del trabajo, San Sebastián, Tercera Prensa.

Veira Veira, J. L. y Muñoz, C. (2004): "Valores y actitudes del trabajo en Europa occidental", en Revista española de Sociología, n. ${ }^{\circ}$, FES, Madrid.

\section{Bases de datos utilizadas}

- Bases de datos (SPSS) de la Encuesta Europea de Valores (Europa 1999 y 2008; España 1999 y 2008; País Vasco 1999 y 2008). 